\title{
Legacy effects of historical grazing alter leaf stomatal characteristics in progeny plants
}

\author{
Jingjing Yin ${ }^{1,2}$, Xiliang $\mathbf{L i}^{2}$, Huiqin Guo ${ }^{3}$, Jize Zhang ${ }^{2}$, Lingqi Kong ${ }^{2}$, Weibo Ren ${ }^{\text {Corresp. } 1}$ \\ ${ }^{1}$ School of Ecology and Environment, Inner Mongolia University, Hohhot, China \\ 2 Institute of grassland Research, Chinese Academy of Agriculture Sciences, Hohhot, China \\ 3 School of Life sciences, Inner Mongolia Agricultural University, Hohhot, China \\ Corresponding Author: Weibo Ren \\ Email address: 111979364@imu.edu.cn
}

Grazing, one of the primary utilization modes of grassland, is the main cause of grassland degradation. Historical overgrazing results in dwarf phenotype and decreased photosynthesis of perennial plants. However, it remains unknown what the mechanism underlying of this legacy effect is, and the role of stomata in the resulting decreased photosynthesis also remains unclear. To address these questions, differences in stomatal density, length and width on both adaxial and abaxial epidermis were compared between overgrazing and ungrazed Leymus chinensis offspring by using rhizome buds cultivated in a greenhouse, and the correlation between photosynthetic capacity and stomatal behavior were also investigated. Our results showed that historical grazing significantly impacted phenotype, photosynthesis and stomatal traits of $L$. chinensis. The offspring plants taken from overgrazed parents were dwarfed compared to those taken from ungrazed parents, and the photosynthesis and stomatal conductance of plants with a grazing history decreased by $28.6 \%$ and $21.3 \%$ respectively. In addition, stomatal density and length on adaxial and abaxial leaf surfaces were significantly increased; however, stomatal width on abaxial leaf surfaces of overgrazed $L$. chinensis was significantly decreased compared with ungrazed individuals. Moreover, the expression patterns of eight genes related to stomatal regulation were tested: seven were down-regulated (2-18 times) and one was up-regulated (three times). Genes, involved in ABC transporter and receptor-like serine/threonine protein kinase were down-regulated. These results suggest that legacy effects of historical grazing affect the stomatal conductance by decreasing the stomatal width in progeny plants, which thus results in lower photosynthesis. Furthermore, changes of stomatal traits and function were regulated by the inhibition of $A B C$ transporter and serine/threonine protein kinase. These findings are helpful for future exploration of the possible mechanisms underlying the response of grassland plants to long-term overgrazing. 


\section{Legacy effects of historical grazing alter leaf stomatal}

\section{2 characteristics in progeny plants}

3 Jingjing Yin ${ }^{1,2}$, Xiliang $\mathrm{Li}^{2}$, Huiqin Guo ${ }^{3}$, Jize Zhang ${ }^{2}$, Lingqi Kong ${ }^{2}$, Weibo Ren ${ }^{1, *}$

$4 \quad{ }^{1}$ School of Ecology and Environment, Inner Mongolia University, Hohhot, China

$5 \quad{ }^{2}$ Institute of Grassland Research, Chinese Academy of Agriculture Sciences, Hohhot, China

$6 \quad{ }^{3}$ School of Life sciences, Inner Mongolia Agriculture University, Hohhot, China

7 * Corresponding author: 111979364@imu.edu.cn

\section{ABSTRACT}

9 Grazing, one of the primary utilization modes of grassland, is the main cause of grassland degradation.

10 Historical overgrazing results in dwarf phenotype and decreased photosynthesis of perennial plants. However,

11 it remains unknown what the mechanism underlying of this legacy effect is, and the role of stomata in the resulting decreased photosynthesis also remains unclear. To address these questions, differences in stomatal density, length and width on both adaxial and abaxial epidermis were compared between overgrazing and ungrazed Leymus chinensis offspring by using rhizome buds cultivated in a greenhouse, and the correlation between photosynthetic capacity and stomatal behavior were also investigated. Our results showed that historical grazing significantly impacted phenotype, photosynthesis and stomatal traits of $L$. chinensis. The offspring plants taken from overgrazed parents were dwarfed compared to those taken from ungrazed parents, and the photosynthesis and stomatal conductance of plants with a grazing history decreased by $28.6 \%$ and $21.3 \%$ respectively. In addition, stomatal density and length on adaxial and abaxial leaf surfaces were significantly increased; however, stomatal width on abaxial leaf surfaces of overgrazed L. chinensis was significantly decreased compared with ungrazed individuals. Moreover, the expression patterns of eight genes related to stomatal regulation were tested: seven were down-regulated (2-18 times) and one was up-regulated (three times). Genes, involved in $\mathrm{ABC}$ transporter and receptor-like serine/threonine protein kinase were downregulated. These results suggest that legacy effects of historical grazing affect the stomatal conductance by decreasing the stomatal width in progeny plants, which thus results in lower photosynthesis. Furthermore, changes of stomatal traits and function were regulated by the inhibition of $\mathrm{ABC}$ transporter and serine/threonine protein kinase. These findings are helpful for future exploration of the possible mechanisms underlying the response of grassland plants to long-term overgrazing.

Keywords Leymus chinensis, Historical grazing, Legacy effects, Stomata, Photosynthesis, Dwarf phenotype 
al., 2009; Schönbach et al., 2011; Vanderpost et al., 2011). Previous studies have also shown that long-term overgrazing can change the composition of plant species from a perennial grass-dominated state to several degraded states dominated by sub-shrubs, shrubs, or annual plants in grasslands (Wang, Wang \& Wang, 2006; Sasaki et al., 2008; Lohmann et al., 2012). Moreover, overgrazing was found to increase soil sand content, while decreasing both the macroporosity and water-holding capacity of soil (Zhao et al., 2011). Recently, it has been suggested that grazing-induced changes in plant traits may persist even after the grazing pressure is removed. This may therefore affect the performance of the following progeny plants, a phenomenon that is called the legacy effect (Veen et al., 2014; Casas et al., 2016).

The legacy effect has been described in ecology since the early 1990s and has been shown to be involved in plant succession, plant invasion, human land-use impacts, and herbivory or grazing impacts (Simard, 1995; Cuddington, 2011). The legacy effect of herbivory, which means that herbivory-induced effects in the parental generation can result in the modification of offspring plants, has recently received increasing attention (Agrawal, Laforsch \& Tollrian, 1999; Poma et al., 2014). Herbivory-induced effects may persist across one or more generations of the offspring via sexual or asexual reproduction (Yin et al., 2019). Generally, these legacy effects are often considered adaptive, particularly when they trigger pre-adaption in offspring plants to similar stresses that parent plants have experienced (Herman \& Sultan, 2011; Holeski, Jander \& Agrawal, 2012). A meta-analysis summarizing 139 experimental studies in plants and animals, focusing on 1170 effect sizes, showed that transgenerational effects benefit offspring plants in response to stressful conditions (Yin et al., 2019). Moreover, legacy effects can be enabled by altering seed quality (Herman \& Sultan, 2011) or by epigenetic modification (Holeski, Jander \& Agrawal, 2012). For example, drought stress has been shown to have negative effects on seed quality by reducing germination and seedling vigor in the F1 generation (Wijewardana et al., 2019). Boyko et al. (2010) showed that stress-induced trans-generational effects were regulated by changes in DNA methylation and small-RNA silencing in Arabidopsis thaliana.

Over the past few decades, much research has been conducted to analyze the ecological and physiological aspects of grazing stresses on natural grasslands (Zhang et al., 2017; Dong et al., 2018; Mueller et al., 2017). Recently, studies on the mechanism of degeneration from individual traits have increased. It has been shown that long-term overgrazing led to the miniaturization of plant individuals, causing e.g., stunted height, shortened and narrowed leaves and similar effects (Dun, Wang \& Liang, 2000). At the same time, physiological traits such as photosynthetic rate also decreased severely after long-term overgrazing, which were still maintained in offspring plants (Ren et al., 2017). However, little is known about how this legacy effect on phenotypic characteristics is maintained. Therefore, this paper explored likely underlying mechanisms based on the stomatal behavior and function.

Stomata, the small pores on the surfaces of leaves and stems, formed by a pair of guard cells, play a critical role in the balance of $\mathrm{CO}_{2}$ uptake for photosynthesis and water loss through transpiration. It is interesting to note that the area of the total stomatal pore may only be $5 \%$ of a plant leaf surface (Willmer \& Fricker, 1996). Moreover, previous studies have suggested that photosynthesis is positively correlated with stomatal conductance (gs) (Cowan, Wong \& Farquhar, 1979; Lawson et al., 2018), and maximum level of gs is dictated by the density and size of stomata (Hetherington \& Woodward, 2003; Franks \& Farquhar, 2007). Stomatal characteristics are affected by many factors, such as $\mathrm{CO}_{2}$ (Woodward \& Kelly, 1995; Sekiya N, 2008), water (Yordanova, Uzunova \& Popova, 2005; Arve et al., 2013; Saradadevi et al., 2017), light (Shimazaki et al., 2007; Mott, 2009), and temperature (Liu et al., 2018; Hill et al., 2014). The correct coordination between 
photosynthesis and stomatal behavior is essential for different plant function. Numerous studies have explored the effects of herbivory on photosynthesis (Chen et al., 2005; Peng et al., 2007; Kerchev et al., 2012). In contrast, little is known about the role of stomata in this process. The genetic regulation of stomatal development and movement was mainly studied in the model plant A. thaliana (Nadeau \& Sack, 2002; Vrablova et al., 2017); however few studies attempted to study this in forage plants. Therefore, this topic is worthy of further study and it is particularly interesting which genes participate in the regulation of the stomata response to overgrazing.

Leymus chinensis is a perennial, rhizomatous clonal grass with high palatability and forage value (Liu, 2006), that largely covers the eastern Eurasian temperate grassland area. As the dominant grass species of the grasslands, L. chinensis plays an important role in the grassland ecosystem. The present study focused on L. chinensis and investigated the effects of historical overgrazing on phenotype, photosynthesis and leaf stomatal characteristics of offspring plants. Previous studies showed that a history of overgrazing can lead to dwarfing in plants and significantly decreased photosynthesis (Ren et al., 2017). However, little is known about the regulating mechanisms of this decrease in photosynthesis. As an important organ of photosynthesis, it is unknown whether stomata play a key role in this process. Therefore, two questions were addressed by this research: (i) Do legacy effects induced by historical grazing change the characteristics of leaf stomata in the following generation? (ii) Which genes take part in the regulation of the stomata response to overgrazing?

\section{METERIALS AND METHODS}

\section{Field site and plant material}

The experimental materials were taken from an artificial grassland located at the field station of the Grassland Research Institute of the Chinese Academy of Agricultural Sciences, located in Hohhot, Inner Mongolia, China

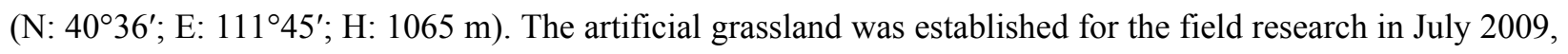
and planted with the forage species L. chinensis, Elymus nutans Griseb., and Medicago sativa L. cv. Aohan., Emanmus sibiricus L., etc. The two treatments included overgrazing (GZ) and no grazing (NG) in this grazing experiment plot, three replications were conducted for each treatment, and the area of each plot was about 0.64 $\mathrm{hm}^{2}$. For grazing treatments, six sheep continuously grazed in the GZ plot during the growing season from May to October each year at a stocking rate of about nine sheep per hectare. This study selected the rhizome buds of $L$. chinensis as offspring generation to examine the legacy effects of historical grazing in parental plants.

\section{Greenhouse experiment}

Fresh grass had not germinated at the early spring of 2018, and the rhizome buds of L. chinensis were randomly taken from both GZ and NG plots in the artificial grassland. The rhizomes coming from different parental plants were cut to 2-3 cm lengths and each rhizome with a bud was planted into a pot and transferred to the greenhouse for cultivation. The buds were kept in the greenhouse for seven weeks. Soil was taken from the grazing experiment plots, and was sieved and mixed to ensure uniformity of the soil. The rhizomes were planted into pots with a diameter of $18 \mathrm{~cm}$ after the pots were filled with soil. Each treatment used seven pots with three rhizomes per pot. In total, 14 pots were randomly arranged in the greenhouse. The temperature was about $25{ }^{\circ} \mathrm{C}$ during the day and $15{ }^{\circ} \mathrm{C}$ at night. The phenotypic traits, gas exchange parameters, stomatal traits, and related gene expression studies were successively tested after plant transplantation based on the following 
methods.

\section{Morphological traits}

The plant height was measured from the $2^{\text {nd }}$ week after transplantation and was continually tested every week. The leaf length (LL) and leaf width (LW) of the second leaf from the top of each individual were measured at week seven of growth in the greenhouse. The leaf area was calculated according to the formula: Area $=0.655^{*}$ (LL $\times$ LW) (Yajun Li, 2009).

\section{Gas exchange measurements}

After about seven weeks of culture, the gas exchange parameters of the second leaf from the top of each plant were measured using a LI-6400 (LI-COR, Lincoln, NE, USA) photosynthetic measurement system during a clear morning from 9:00 to 11:00. Measurement indicators included: net photosynthetic rate $\left(P_{N}\right)$, transpiration rate $(E)$, inter-cellular $\mathrm{CO}_{2}$ concentration $(\mathrm{Ci})$, stomatal conductance $(\mathrm{gs})$, and water use efficiency (WUE) calculated from photosynthetic rate and transpiration rate $\left(W U E=P_{N} / E\right)$. The gas exchanges were measured as photosynthetically active radiation $(P A R)$ of $1000 \pm 10 \mu \mathrm{mol} \mathrm{m} \mathrm{m}^{-2} \mathrm{~s}^{-1}$ and $\mathrm{CO}_{2}$ concentration of $350 \pm 3 \mathrm{ppm}$.

\section{Stomatal traits}

After about seven weeks of culturing, the second leaf of each of the treated plants of L. chinensis was collected to determine the stomatal traits using the impression approach $(X u \& Z h o u, 2008)$. After collection, the leaves were rapidly fixed with $2.5 \%$ glutaraldehyde, and brought back to the laboratory. The abaxial and adaxial epidermis of the leaf were first cleaned using a paper towel, and then carefully smeared with transparent nail polish in the tail, middle, and top area, gently peeled off after approximately $15 \mathrm{~min}$, then immediately mounted on a glass slide, covered with a cover slip, and finally observed and photographed using an optical microscope (BX53, Olympus, Tokyo, Japan). To count the number of stomata in adaxial and abaxial leaf surfaces in the microscope at $40 \times$ magnification, and to calculate the stomatal density (stomatal density $=$ number of stomata / area in view), five fields of view were randomly observed for each piece. Seven stomata were randomly selected from the microscopic slides, and stomatal length and width in the microphotographs were analyzed with Image J 1.0 image processing software (National Institutes of Health, Bethesda, MD, USA). Since the degree of opening of the pores changes constantly, the length and width of guard cells were selected to represent the maximum degree of opening of the pores, i.e., the size of the pores (Supplementary Figure S1).

\section{Real-time PCR analysis}

Samples were taken from the second or third leaf of offspring plants treated with enclosure and overgrazing of parental plants after about seven weeks of culture, then stored at $-80^{\circ} \mathrm{C}$. RNA isolation used the TRIZOL reagent (Invitrogen, USA) following the procedures provided by the manufacturer. Then, the quantity of RNA was evaluated by electrophoresis on a $1.0 \%$ agarose gel, and the concentration was measured by an ultraviolet spectrophotometer (UV-2550PC, Shimadzu, Japan). High-quality total RNA samples were used for subsequent experiments. According to the manufacturer's instructions, any possible DNA contamination was eliminated from RNA samples, and first-stand cDNA was synthesized by using the Prime Script ${ }^{\mathrm{RT}}$ reagent Kit with gDNA Eraser (Takara, Japan). The cDNA samples were stored at $-20{ }^{\circ} \mathrm{C}$ for real-time PCR analysis. In addition, eight differentially expressed genes related to stomatal development and regulation were selected based on transcriptome data (Ren et al., 2018). The program primer premier 5 was used to design the required 
154

155

156

157

158

159

160

161

162

163

164

165

166

167

168

169

170

171

172

173

174

175

176

177

178

179

180

181

182

183

184

185

186

187

188

primer sequences, and the primer details of these genes are presented in Table S1 of the Supporting Information. The real-time PCR for gene expression was performed on Quant Studio ${ }^{\mathrm{TM}} 6$ Flex System Software using SYBR Green 1 reagents, following the instructions of test kits.

\section{Statistical analyses}

All statistical analyses were conducted in Microsoft Excel, and independent samples $\mathrm{T}$ tests were performed to assess the differences between treatments for phenotypic characteristics, photosynthetic parameters, and stomatal traits at the $\mathrm{P}<0.01$ and $\mathrm{P}<0.05$ levels of significance using SPSS 19.0 statistical software (Systat Software Inc., Chicago, IL, USA). Graphical representations were generated with Sigmaplot 13.0 software (Systat Software Inc., San Jose, CA, USA).

\section{RESULTS}

Historical grazing on parent plants significantly affected the growth of offspring plants (Figs. 1, 2). Offspring developed from grazed parents grew much slower than those from ungrazed parents (Fig. 1B). Compared with the ungrazed control, the plant height and leaf length of grazed progeny decreased by $23.5 \%$ and $15.4 \%$ (Figs. $1,2 \mathrm{~A}$ ), the leaf width and area of grazed progeny decreased by $9.7 \%$ and $23.1 \%$ respectively (Figs. $2 \mathrm{~B}, \mathrm{C}$ ), and the aboveground biomass of individual grazed plants also decreased by $12.9 \%$ (Fig. 2D).

Historical grazing has also significant impact on photosynthesis. $P_{N}$ and $g s$ in progeny plants generated from grazed parents were significantly decreased by $28.6 \%$ and $21.3 \%$, respectively, compared with control plants $(\mathrm{P}<0.05$, Figs. 3A, B). However, $C i$ in ungrazed progeny plants increased and $W U E$ decreased significantly $(\mathrm{P}<0.05$, Figs. 3C, E).

Stomatal density on the basal adaxial leaf surfaces in progeny plants of grazing-stressed L. chinensis increased significantly in the greenhouse experiment (Fig. 4A). However, no significant differences were found both in the middle and top leaf surfaces (Figs. 4B, C). Noticeably, historical grazing significantly increased stomatal density and length; however, the stomatal width on the basal of abaxial leaf surfaces decreased (Figs. 4D, E, F). Moreover, stomatal density and width on the middle of abaxial leaf surfaces in offspring plants taken from grazed parents decreased significantly compared with those taken from ungrazed parents (Figs. 4D, E, F). Notably, no significant differences were found in stomatal characteristics on the top of adaxial and abaxial leaf surfaces (Figs. 4D, E, F), suggesting that stomatal differences were mainly found at the basal and middle of leaves, not at the top of leaves.

To explore the possible mechanism of stomatal differences induced by historical grazing, the expressions of eight key genes related to stomatal regulation were investigated (Table 1). These genes are classified into two types based on the pathways they are involved in: 1) stomatal movements (ABCCL4, ABCG5, SAPK10, and GNAT1); 2) stomatal development (ER1, ER, ERL1, and CYCP3L-1) (Table 1). The results showed that all genes of grazed progeny plants were down-regulated by 2-18 times except for $C Y C P 3 L-1$ which was upregulated (Figs. 5A-H). This suggests that stomatal characteristics were mainly affected by the downregulation of related genes. 
190

191

192

193

194

195

196

197

198

199

200

201

202

203

204

205

206

207

208

209

210

211

212

213

214

215

216

217

218

219

220

221

222

223

224

225

226

227

\section{The effects of historical grazing on stomatal characteristics}

Plants can alter stomatal activity to cope with environmental stresses such as drought (Changhai et al., 2010), cold (Reynolds-Henne et al., 2010), insects (Papazian et al., 2016), and even grazing (Zhang Lr, 2010). This study provides clear evidence that the grazing history can impress legacy effects on traits and functions of leaf stomata in L.chinensis. After grazing, stomatal density increased significantly on the basal of adaxial and abaxial leaf surfaces and decreased significantly on the middle of abaxial leaf surfaces. Similar findings have been reported for Thalictrum aplinum, Kobresia humilis, Gentiana straminea, and Elymus nutans Griseb (Zhang Lr, 2010). Moreover, stomatal length of the progeny developed from grazed parents increased, while stomatal width decreased significantly in abaxial leaf surfaces. These data suggested that stomata of progeny plants tended to close because of the long-term overgrazing experience of their parental plants, which resulted in a decrease in gs. A similar result of stomatal closure caused by herbivory was also reported previously (Papazian et al., 2016). Hence, it may be an alternative strategy to close the stomata to adapt to grazing stress. Stomata are deliberately regulated by a series of genes to help plants adapt to environmental stimuli. This study investigated eight key genes that play key roles in the development and movement of stomata. It has been suggested that historical grazing affects the expression level of these genes on the progeny developed from grazed parents. After long-term grazing, the expression of seven of these eight tested genes, mainly related to stomatal development and regulation, were significantly decreased. For example, $A B C C L 4$ and $A B C G 5$ are two members in the superfamily of the ATP-binding cassette (ABC) transporters, which has more than 120 members in both rice (Oryza sativa) and A. thaliana. Stomatal regulation is one of the fundamental processes in which $\mathrm{ABC}$ transporters participate (Rea, 2007). A previous study supported that $\mathrm{ABC}$ transporter AtMRP 5 mutants tended to have more closed stomata than wild-type control plants, resulting in decreased transpiration rate and increased drought tolerance (Klein et al., 2003). Therefore, the down-regulation of the expressions of $A B C C L 4$ and $A B C G 5$ may also be relevant to closing stomata. In addition, GNAT1 plays an important role in signal transmission, as $\mathrm{G}$ proteins participate in ethylene-induced stomatal closure and functions through hydrogen peroxide synthesis in A. thaliana (Ge et al., 2015). Moreover, in this study, three cell-surface-resident receptor-like kinases genes (ER1, $E R$, and $E R L 1)$ were detected, which encode members of the large family of plant LRR receptor-like kinases (RLKs) (Shpak et al., 2004), which play a critical role in the regulation of stomatal density. Shpak (2005) identified the ER-family LRR-RLKs as negative regulators of stomatal development, and the down-regulation of $E R$ genes and the up-regulation of $C Y C P 3 L-1$ provide molecular evidence for increased stomatal density and length on the basal of adaxial and abaxial leaf surfaces. Another serine / threonine - protein kinase gene (SAPK10), which is specifically expressed in guard cells, plays an important role in stomatal movement (Min et al., 2019). However, the regulation mechanism of SAPK10 remains unclear and requires further study.

\section{Legacy effects on stomata}

Stomatal numbers and their pattern on the leaf surface can be either directly or indirectly influenced by the growth environment. Stomatal density is one of the key factors that may regulate the stomatal conductance and functions (Doheny-Adams et al., 2012). Increased stomatal density offers more potential for $\mathrm{CO}_{2}$ diffusion into the leaf, which facilitates higher rates of gas exchange in responses to environmental stimuli (Yoo et al., 2011;

PeerJ reviewing PDF | (2019:12:44172:1:1:NEW 19 Apr 2020) 
de Boer et al., 2016). Furthermore, a close positive correlation exists between stomatal conductance and photosynthetic rate (Buckley \& Mott, 2013; Sharkey, 1982). Tanaka et al. (2013) investigated A. thaliana mutants with altered stomatal density and showed that the photosynthetic rate could be enhanced by $30 \%$ via increasing stomatal density in STOMAGEN-overexpressing A. thaliana compared with wild-type plants. In the present research, the photosynthetic rate decreased significantly in response to long-term grazing treatment in the parental generation of L. chinensis. A similar finding was also reported in previous research (Ren et al., 2017). The performance is the same in the subsequent generation that developed from grazed parents. However, the photosynthetic rate of offspring plants decreased by $28.6 \%$ compared with that of the un-grazed control. The possible reason is that stomatal aperture weights much than stomatal density on which gs is determined (Weyers \& Lawson, 1997). Furthermore, changes in stomatal density can be compensated for by changes in stomatal opening or closure. For example, Büessis et al. (2006) used transgenic $A$. thaliana plants that overexpressed the $S D D 1$ gene and $s d d 1-1$ mutants showed that increased stomatal density could be compensated for by decreased stomatal aperture.

Many studies have showed that livestock grazing may change soil quality or properties and leads to soil compaction relative to ungrazed soil (Daniel, 2002; Drewry J J, 2008; Kotze et al., 2013; Manuel Pulido, 2016; Byrnes et al., 2018). This in turn can result in decreased soil pore space, reduced infiltration, and even decrease the supply of available water (Willatt \& Pullar, 1984; Kotze et al., 2013; Kenneth W. Tate et al., 2004). To address the water limitation, plants may decrease their stomatal aperture to minimize water loss. However, the closure of stomata may also decrease the uptake of $\mathrm{CO}_{2}$, which may decrease the photosynthetic rate and inhibit plant growth. Hence, plants may face the trade-off between water loss and $\mathrm{CO}_{2}$ uptake. For example, Case \& Barrett (2001) investigated two populations of Wurmbea dioica separately in wet and dry conditions, their results showed that the stomata of the dry populations were partly closed to minimize water loss and decrease photosynthesis rates. The detailed mechanisms of the stomatal response to grazing are not easy to explain, because grazing involves a complex set of factors. However, the results of the present study suggested the existence of legacy effects of livestock grazing in the parents with regard to the stomatal behavior in offspring generation of L. chinensis. Moreover, the changes in stomatal characteristics decreased stomatal conductance and photosynthesis rate, which resulted in a dwarf phenotype of offspring plants (Fig. 6). So far, exceedingly little is known about the detailed mechanisms of these legacy effects; however, increasing evidence suggests that epigenetic mechanisms such as DNA methylation, histone modifications, and small RNAs contribute to these legacy effects (Agrawal, 2001; Champagne, 2008; Richards, 2011; Boyko et al., 2010). Further research is needed to fully understand the epigenetic mechanisms of how the legacy effects are induced by grazing from the parents to progeny plants.

\section{Legacy effects of historical grazing on progeny plants}

Long-term overgrazing, which can affect the stability of the plant community by altering the growth of individual plants (Niu et al., 2015; Zuo et al., 2018), is an important cause for grassland degradation (Bai et al., 2012). Previous research has shown that transgenerational morphological plasticity induced by overgrazing heavily involves photosynthetic function in L. chinensis (Ren et al., 2017). In general, phenotypic plasticity refers to the ability of organisms to modify their morphology, physiology, or behavior and adapt to changing environmental conditions (Wadgymar \& Austen, 2019). This experiment further observed that overgrazing experienced by the parental generation could alter stomatal characteristics of the offspring of L. chinensis. The decreased photosynthesis and the dwarfed phenotype may be attributed to transgenerational stomatal plasticity. 
269

270

271

272

273

274

275

276

277

278

279

280

281

282

283

284

285

286

287

288

289

290

291

292

293

294

295

296

297

298

299

300

301

302

303 6

However, little is known about transgenerational effects induced by overgrazing in forage grass. According to a recent study by Kafle \& Wurst (2019), single herbivory in the parental generation did not prime the transgenerational response, only sequential above- and below- ground herbivory did. Therefore, it can be speculated that transgenerational responses require a certain threshold of environmental stress. Moreover, the developmental stage at which the parental generation is subjected to stress can also influence the performance of their offspring (Burton \& Metcalfe, 2014). However, for perennial asexual plants, the threshold of crossgenerational effects and how long it can last in the offspring generation remain unknown and require further exploration.

Transgenerational legacy effects have received increasing attention in recent years. Although only the legacy effects raised from historical grazing in L. chinensis was investigated in this study, the results still provide hints what may happen in other plants. Holeski, Jander \& Agrawal (2012) have also summarized several examples of transgenerational effects of the resistance to herbivores and pathogens in 11 plant species. Previous studies have shown that the offspring generation could perform better in the maternal than in the nonmaternal environment (Galloway \& Etterson, 2007; Latzel et al., 2014). L. chinensis, as an asexual plant, consists of many interconnected ramets, where each ramet may experience different environmental conditions. This leads to better adaptation of offspring ramets to the living environment by inheriting information from the maternal generation. Therefore, the legacy effects of historical grazing are more conducive to both the survival and growth of progeny plants.

\section{CONCLUSION}

This study found that legacy effects of historical grazing can alter leaf stomatal characteristics in progeny plants. The results suggested the existence of a trade-off between density and aperture of stomata in $L$. chinensis in response to long-term overgrazing stress. Changes in stomatal characteristics caused decreases in gs and photosynthesis rate, which resulted in a dwarf phenotype of offspring plants. Moreover, five important genes ( $A B C C L, A B C G 5, E R 1, E R$, and $E R L 1)$, which are involved in $\mathrm{ABC}$ transporter and receptor-like serine/threonine protein kinase, played a key role in the process of stomatal regulation. This regulation mechanism of the process has important significance for a better understanding of the dwarf phenotype of perennial grassland plants.

\section{ACKNOWLEDGEMENTS}

We gratefully thank Fenghui Guo for his help with fieldwork, and we also thank Xiumin Yu for the technical help during the experiment.

\section{ADDITIONAL INFORMATION AND DECLARATIONS}

\section{Funding}

This project was supported by the National Natural Science Foundation of China (No. 31872407). The funders had no role in study design, data collection and analysis, decision to publish, or preparation of the manuscript. 


\section{Grant Disclosures}

305 The following grant information was disclosed by the authors: 31872407 .

\section{Competing Interests}

307 The authors declare there are no competing interests.

\section{Author Contributions}

309 Jingjing Yin conceived and designed the experiments, performed the experiments, contributed

310 reagents/materials/analysis tools, analyzed the date, prepared figures and/or tables, authored or reviewed drafts 311 of the paper, approved the final draft.

312 Xiliang Li designed the experiments, analyzed the date, authored or reviewed drafts of the paper.

313 Huiqin Guo, Jize Zhang and Lingqi Kong contributed reagents/materials/analysis tools, authored or reviewed 314 drafts of the paper.

315 Weibo Ren designed the experiments, prepared figures and/or tables, authored or reviewed drafts of the paper, 316 approved the final draft. 
317

318

319

320

321

322

323

324

325

326

327

328

329

330

331

332

333

334

335

336

337

338

339

340

341

342

343

344

345

346

347

348

349

350

351

352

353

354

355

356

\section{REFERENCES}

Agrawal. 2001. Transgenerational consequences of plant responses to herbivory: an adaptive maternal effect? American Naturalist 157:555-569 DOI 10.1086/319932.

Agrawal, Laforsch, Tollrian. 1999. Transgenerational induction of defences in animals and plants. Nature 401:60-63 DOI 10.1038/43425.

Arve, Terfa, Gislerod, Olsen, Torre. 2013. High relative air humidity and continuous light reduce stomata functionality by affecting the aba regulation in rose leaves. Plant Cell and Environment 36:382-392 DOI 10.1111/j.1365-3040.2012.02580.x.

Bai, Wu, Clark, Pan, Zhang, Chen, Wang, Han. 2012. Grazing alters ecosystem functioning and C:N:P stoichiometry of grasslands along a regional precipitation gradient. Journal of Applied Ecology 49:12041215 DOI 10.1111/j.1365-2664.2012.02205.x.

Boer, Price, Wagner-Cremer, Dekker, Franks, Veneklaas. 2016. Optimal allocation of leaf epidermal area for gas exchange. New Phytologist 210:1219-1228 DOI 10.1111/nph.13929.

Boyko, Blevins, Yao, Golubov, Bilichak, Ilnytskyy, Hollander, Jr. Meins, Kovalchuk. 2010. Transgenerational adaptation of Arabidopsis to stress requires DNA methylation and the function of dicerlike proteins. Plos One 5:e9514 DOI 10.1371/journal.pone.0009514.

Buckley, Mott. 2013. Modelling stomatal conductance in response to environmental factors. Plant, Cell \& Environment 36:1691-1699 DOI 10.1111/pce.12140.

Büessis, von Groll, Fisahn, Altmann. 2006. Stomatal aperture can compensate altered stomatal density in Arabidopsis thaliana at growth light conditions. Functional Plant Biology 33:1037-1043 DOI 10.1071/FP06078.

Burton, Metcalfe. 2014. Can environmental conditions experienced in early life influence future generations? Proceedings of the Royal Society B-Biological Sciences 281 DOI 10.1098/rspb.2014.0311.

Byrnes, Eastburn, Tate, Roche. 2018. A global meta-analysis of grazing impacts on soil health indicators. Journal of Environmental Quality 47:758-765 DOI 10.2134/jeq2017.08.0313.

Casas, Torretta, Exeler, Omacini. 2016. What happens next? Legacy effects induced by grazing and grassendophyte symbiosis on thistle plants and their floral visitors. Plant and Soil 405:211-229 DOI 10.1007/s11104-015-2644-9.

Case, Barrett. 2001. Ecological differentiation of combined and separate sexes of Wurmbea dioica (colchicaceae) in sympatry. Ecology 82:2601-2616 DOI 10.1890/00129658(2001)082[2601:EDOCAS]2.0.CO;2.

Champagne. 2008. Epigenetic mechanisms and the transgenerational effects of maternal care. Frontiers in Neuroendocrinology 29:386-397 DOI 10.1016/j.yfrne.2008.03.003.

Changhai, Baodi, Yunzhou, Yuxin, Lei, Mengyu, Haipei. 2010. Physiological regulation of high transpiration efficiency in winter wheat under drought conditions. Plant, Soil and Environment 56:340-347 DOI 10.17221/220/2009-PSE.

Chen, Bai, Lin, Liang, Han. 2005. Effects of grazing on photosynthetic characteristics of major steppe species in the Xilin river basin, Inner Mongolia, China. Photosynthetica 43:559-565 DOI 10.1007/s11099005-0088-9.

Cowan, Wong, Farquhar. 1979. Stomatal conductance correlates with photosynthetic capacity. Nature 
282:424-426 DOI 10.1038/282424a0.

Cuddington. 2011. Legacy effects: the persistent impact of ecological interactions. Biological Theory 6:203210 DOI 10.1007/s13752-012-0027-5.

Daniel. 2002. Long-term grazing density impacts on soil compaction. Transactions of the ASAE 45:1911 DOI $10.13031 / 2013.11442$.

Doheny-Adams, Hunt, Franks, Beerling, Gray. 2012. Genetic manipulation of stomatal density influences stomatal size, plant growth and tolerance to restricted water supply across a growth carbon dioxide gradient. Philosophical Transactions of the Royal Society B: Biological Sciences 367:547-555 DOI 10.1098/rstb.2011.0272.

Dong, Wang, Liu, Luo, Li, Yu. 2018. Direct and legacy effects of herbivory on growth and physiology of a clonal plant. Biological Invasions 20:3631-3645 DOI 10.1007/s10530-018-1801-5.

Drewry J J. 2008. pasture yield and soil physical property responses to soil compaction from treading and grazing - a review. Australian Journal of Soil Research 46:237-256 DOI 10.1071/SR07125.

Dun, Wang, Liang. 2000. Analysis of the plant individual behaviour during the degradation and restoring succession in steppe community. Acta Phytoecolog ica Sinica 24:268-274.

Franks, Farquhar. 2007. The mechanical diversity of stomata and its significance in gas-exchange control. Plant Physiology 143:78-87 DOI 10.1104/pp.106.089367.

Galloway, Etterson. 2007. Transgenerational plasticity is adaptive in the wild. Science 318:1134-1136 DOI 10.1126/science.1148766.

Ge, Cai, Lei, Zhou, Yue, He. 2015. Heterotrimeric g protein mediates ethylene-induced stomatal closure via hydrogen peroxide synthesis in Arabidopsis. The Plant Journal 82:138-150 DOI 10.1111/tpj.12799.

Herman, Sultan. 2011. Adaptive transgenerational plasticity in plants: case studies, mechanisms, and implications for natural populations. Frontiers in Plant Science 2 DOI 10.3389/fpls.2011.00102.

Hetherington, Woodward. 2003. The role of stomata in sensing and driving environmental change. Nature 424:901-908 DOI 10.1038/nature01843.

Hill, Guerin, Hill, Watling. 2014. Temperature influences stomatal density and maximum potential water loss through stomata of Dodonaea viscosa subsp. angustissima along a latitude gradient in southern Australia. Australian Journal of Botany 62:657 DOI 10.1071/BT14204.

Holeski, Jander, Agrawal. 2012. Transgenerational defense induction and epigenetic inheritance in plants. Trends in Ecology \& Evolution 27:618-626 DOI 10.1016/j.tree.2012.07.011.

Kafle, Wurst. 2019. Legacy effects of herbivory enhance performance and resistance of progeny plants. Journal of Ecology 107:58-68 DOI 10.1111/1365-2745.13038.

Kenneth W. Tate, Dennis M Dudley, Neil K. Mcdougald, George. 2004. effect of canopy and grazing on soil bulk density. Journal of Range Management 57:411-417 DOI 10.2307/4003867.

Kerchev, Fenton, Foyer, Hancock. 2012. Plant responses to insect herbivory: interactions between photosynthesis, reactive oxygen species and hormonal signalling pathways. Plant Cell and Environment 35:441-453 DOI 10.1111/j.1365-3040.2011.02399.x.

Klein, Perfus Barbeoch, Frelet, Gaedeke, Reinhardt, Mueller Roeber, Martinoia, Forestier. 2003. The plant multidrug resistance ABC transporter AtMRP5 is involved in guard cell hormonal signalling and water use. The Plant Journal 33:119-129 DOI 10.1046/j.1365-313X.2003.016012.x.

Kotze, Sandhage-Hofmann, Meinel, du Preez, Amelung. 2013. Rangeland management impacts on the 
398

399

400

401

402

403

404

405

406

407

408

409

410

411

412

413

414

415

416

417

418

419

420

421

422

423

424

425

426

427

428

429

430

431

432

433

434

435

436

437

438

properties of clayey soils along grazing gradients in the semi-arid grassland biome of South Africa. Journal of Arid Environments 97:220-229 DOI 10.1016/j.jaridenv.2013.07.004.

Krzic, Newman, Trethewey, Bulmer, Chapman. 2006. Cattle grazing effects on plant species composition and soil compaction on rehabilitated forest landings in central interior British Columbia. Journal of Soil and Water Conservation 61:137.

Latzel, Janeček, Doležal, Klimešová, Bossdorf. 2014. Adaptive transgenerational plasticity in the perennial Plantago lanceolata. Oikos 123:41-46 DOI 10.1111/j.1600-0706.2013.00537.x.

Lawson, Terashima, Fujita, Wang. 2018. Coordination between photosynthesis and stomatal behavior. In: Adams III, W.W., Terashima, I., eds. The Leaf: A Platform for Performing Photosynthesis. Springer International Publishing: Cham, 141-161.

Liang, Han, Zhou, Zhao, Snyman, Shan, Havstad. 2009. Grazing intensity on vegetation dynamics of a typical steppe in northeast Inner Mongolia. Rangeland Ecology \& Management 62:328-336 DOI https://doi.org/10.2111/08-167.1.

Liu. 2006. Effects of sowing depth on the seedling emergence of Leymus chinensis with different seed source. Seed 25:20-23.

Liu, He, Zhang, Li, Wang, Sack, Yu. 2018. Variation of stomatal traits from cold temperate to tropical forests and association with water use efficiency. Functional Ecology 32:20-28 DOI 10.1111/13652435.12973.

Lohmann, Tietjen, Blaum, Joubert, Jeltsch. 2012. Shifting thresholds and changing degradation patterns: climate change effects on the simulated long-term response of a semi-arid savanna to grazing. Journal of Applied Ecology 49:814-823 DOI 10.1111/j.1365-2664.2012.02157.x.

Manuel Pulido. 2016. The impact of heavy grazing on soil quality and pasture production in rangelands of SW Spain. Land Degradation \& Development 29:219-230 DOI 10.1002/ldr.2501.

Min, Choi, Kim, Yoon, Han, Lee, Lee, Kim. 2019. Two clade a phosphatase 2 cs expressed in guard cells physically interact with abscisic acid signaling components to induce stomatal closure in rice. Rice 12 DOI 10.1186/s12284-019-0297-7.

Mott. 2009. Opinion: stomatal responses to light and $\mathrm{CO}_{2}$ depend on the mesophyllpce. Plant, Cell \& Environment 32:1479-1486 DOI 10.1111/j.1365-3040.2009.02022.x.

Mueller, Granse, Nolte, Hai, Weingartner, Hoth, Jensen. 2017. Top-down control of carbon sequestration: grazing affects microbial structure and function in salt marsh soils. Ecological Applications 27:1435-1450 DOI 10.1002/eap.1534/full.

Nadeau, J.A., Sack, F.D. 2002. Control of stomatal distribution on the Arabidopsis leaf surface. Science 296:1697-1700 DOI 10.1126/science.1069596.

Niu, Messier, He, Lechowicz. 2015. The effects of grazing on foliar trait diversity and niche differentiation in Tibetan alpine meadows. Ecosphere 6:150 DOI 10.1890/ES14-00547.1.

Papazian, Khaling, Bonnet, Lassueur, Reymond, Moritz, Blande, Albrectsen. 2016. Central metabolic responses to ozone and herbivory affect photosynthesis and stomatal closure1[open]. Plant Physiology 172:2057-2078 DOI 10.1104/pp.16.01318.

Peng, Jiang, Liu, Niu, Liu, Biswas. 2007. Photosynthesis, transpiration and water use efficiency of four plant species with grazing intensities in Hunshandak Sandland, China. Journal of Arid Environments 70:304315 DOI https://doi.org/10.1016/j.jaridenv.2007.01.002.

PeerJ reviewing PDF | (2019:12:44172:1:1:NEW 19 Apr 2020) 
439

440

441

442

443

444

445

446

447

448

449

450

451

452

453

454

455

456

457

458

459

460

461

462

463

464

465

466

467

468

469

470

471

472

473

474

475

476

477

478

479

Poma, Colafarina, Fontecchio, Chichiricco. 2014. Transgenerational effects of nms. In: Capco, D.G., Chen, Y., eds. Advances in Experimental Medicine and Biology, 235-254.

Rea. 2007. Plant ATP-binding cassette transporters. Annual Review of Plant Biology 58:347-375 DOI 10.1146/annurev.arplant.57.032905.105406.

Ren, Hu, Hou, Zhang, Guo, Liu, Kong, Wu, Wang, Li. 2017. Long-term overgrazing-induced memory decreases photosynthesis of clonal offspring in a perennial grassland plant. Frontiers in Plant Science $\mathbf{8}$ DOI 10.3389/fpls.2017.00419.

Ren, Xie, Hou, Li, Guo, Hu, Kong, Zhang, Chang, Wu. 2018. Potential molecular mechanisms of overgrazing-induced dwarfism in sheepgrass (Leymus chinensis) analyzed using proteomic data. Bmc Plant Biology 18:81 DOI 10.1186/s12870-018-1304-7.

Reynolds-Henne, Langenegger, Mani, Schenk, Zumsteg, Feller. 2010. Interactions between temperature, drought and stomatal opening in Legumes. Environmental and Experimental Botany 68:37-43 DOI 10.1016/j.envexpbot.2009.11.002.

Richards. 2011. Natural epigenetic variation in plant species: a view from the field. Current Opinion in Plant Biology 14:204-209 DOI https://doi.org/10.1016/j.pbi.2011.03.009.

Saradadevi, Bramley, Palta, Siddique. 2017. Stomatal behaviour under terminal drought affects postanthesis water use in wheat. Functional Plant Biology 44:279 DOI 10.1071/FP16078.

Sasaki, Okayasu, Jamsran, Takeuchi. 2008. Threshold changes in vegetation along a grazing gradient in Mongolian rangelands. Journal of Ecology 96:145-154 DOI 10.1111/j.1365-2745.2007.01315.x.

Schönbach, Wan, Gierus, Bai, Müller, Lin, Susenbeth, Taube. 2011. Grassland responses to grazing: effects of grazing intensity and management system in an Inner Mongolian steppe ecosystem. Plant and Soil 340:103-115 DOI 10.1007/s11104-010-0366-6.

Sekiya N. 2008. Stomatal density of cowpea correlates with carbon isotope discrimination in different phosphorus, water and $\mathrm{CO}_{2}$ environments. New Phytologist 179:799-807 DOI 10.1111/j.14698137.2008.02518.x.

Sharkey. 1982. Stomatal conductance and photosynthesis. DOI 10.1111/nph.16142.

Shimazaki, Doi, Assmann, Kinoshita. 2007. Light regulation of stomatal movement. Annual Review of Plant Biology 58:219-247 DOI 10.1146/annurev.arplant.57.032905.105434.

Shpak. 2005. Stomatal patterning and differentiation by synergistic interactions of receptor kinases. Science 309:290-293 DOI 10.1126/science.1109710.

Shpak, Berthiaume, Hill, Torii. 2004. Synergistic interaction of three ERECTR-family receptor-like kinases controls Arabidopsis organ growth and flower development by promoting cell proliferation. Development 131:1491-1501 DOI 10.1242/dev.01028.

Simard. 1995. Interspecific carbon transfer in ectomycorrhizal tree species mixtures. ProQuest Dissertations Publishing.

Tanaka, Sugano, Shimada, Hara-Nishimura. 2013. Enhancement of leaf photosynthetic capacity through increased stomatal density in Arabidopsis. New Phytologist 198:757-764 DOI 10.1111/nph.12186.

van der Westhuizen, Snyman, Fouché. 2005. A degradation gradient for the assessment of rangeland condition of a semi-arid sourveld in Southern Africa. African Journal of Range \& Forage Science 22:4758 DOI 10.2989/10220110509485861.

Vanderpost, Ringrose, Matheson, Arntzen. 2011. Satellite based long-term assessment of rangeland 
480

481

482

483

484

485

486

487

488

489

490

491

492

493

494

495

496

497

498

499

500

501

502

503

504

505

506

507

508

509

510

511

512

513

514

515

516

517

518

519

520

condition in semi-arid areas: an example from Botswana. Journal of Arid Environments 75:383-389 DOI https://doi.org/10.1016/j.jaridenv.2010.11.002.

Veen, de Vries, Bakker, van der Putten, Olff. 2014. Grazing-induced changes in plant-soil feedback alter plant biomass allocation. Oikos 123:800-806 DOI 10.1111/j.1600-0706.2013.01077.x.

Vrablova, Vrabl, Hronkova, Kubasek, Santrucek. 2017. Stomatal function, density and pattern, and $\mathrm{CO}_{2}$ assimilation in Arabidopsis thaliana tmm1 and sdd1-1 mutants. Plant Biol (Stuttg) 19:689-701 DOI 10.1111/plb.12577.

Wadgymar, S.M., Austen, E.J. 2019. Shifting perspectives on the impacts of phenotypic plasticity. New Phytologist 224:1009-1011 DOI 10.1111/nph.16210.

Wang W Y, Wang Q J, Wang H C. 2006. The effect of land management on plant community composition, species diversity, and productivity of alpine Kobersia steppe meadow. Ecological Research 21:181-187 DOI 10.1007/s11284-005-0108-z.

Weyers, Lawson. 1997. Heterogeneity in stomatal characteristics. In: Callow, J.A., ed. Advances in Botanical Research. Journal of Experimental Botany, 317-352.

Wijewardana, Reddy, Krutz, Gao, Bellaloui. 2019. Drought stress has transgenerational effects on soybean seed germination and seedling vigor. Plos One 14:e214977 DOI 10.1371/journal.pone.0214977.

Willatt, Pullar. 1984. Changes in soil physical properties under grazed pastures. Soil Research 22:343-348.

Willmer, Fricker. 1996. Stomata. Springer Science \& Business Media.

WOODWARD, KELLY. 1995. The influence of $\mathrm{CO}_{2}$ concentration on stomatal density. New Phytologist 131:311-327 DOI 10.1111/j.1469-8137.1995.tb03067.x.

$\mathbf{X u}$, Zhou. 2008. Responses of leaf stomatal density to water status and its relationship with photosynthesis in a grass. Journal of Experimental Botany 59:3317-3325 DOI 10.1093/jxb/ern185.

Yajun Li. 2009. The determination method of leaf area on Leymus chinensis. Journal of Anhui Agri.Sci 37:6819-6851 DOI 10.13989/j.cnki.0517-6611.2009.15.093.

Yin, Zhou, Lin, Li, Zhang. 2019. Transgenerational effects benefit offspring across diverse environments: a meta - analysis in plants and animals. Ecology Letters DOI 10.1111/ele.13373.

Yoo, Pence, Jin, Miura, Gosney, Hasegawa, Mickelbart. 2011. The Arabidopsis GTL1 transcription factor regulates water use efficiency and drought tolerance by modulating stomatal density via transrepression of SDD1. The Plant Cell 22:4128-4141 DOI 10.1105/tpc.110.078691.

Yordanova, Uzunova, Popova. 2005. Effects of short-term soil flooding on stomata behaviour and leaf gas exchange in barley plants. Biologia Plantarum 49:317-319 DOI 10.1007/s10535-005-7319-6.

Zhang LR. 2010. Effects of temperature increase and grazing on stomatal density and length of four alpine kobresia meadow species, Qinghai-tibetan plateau. Acta Ecologica Sinica 30:6961-6969.

Zhang, Zuo, Zhou, Lv, Lian, Yue. 2017. Long-term grazing effects on vegetation characteristics and soil properties in a semiarid grassland, northern China. Environmental Monitoring and Assessment 189 DOI 10.1007/s10661-017-5947-x.

Zhao, Peth, Reszkowska, Gan, Krümmelbein, Peng, Horn. 2011. Response of soil moisture and temperature to grazing intensity in a Leymus chinensis steppe, Inner Mongolia. Plant and Soil 340:89-102 DOI 10.1007/s11104-010-0460-9.

Zuo, Zhang, Lv, Wang, Yang, Yue, Zhou, Li, Chen, Lian, Qu, Liu, Ma. 2018. Effects of plant functional diversity induced by grazing and soil properties on above- and below ground biomass in a semiarid

PeerJ reviewing PDF | (2019:12:44172:1:1:NEW 19 Apr 2020) 
521 grassland. Ecological Indicators 93:555-561 DOI 10.1016/j.ecolind.2018.05.03. 
Figure 1

The differences in growth of offspring plants developed from overgrazed and ungrazed Leymus chinensis respectively in the culture period (Mean \pm SE).

NG, Ungrazed; GZ, Grazed.
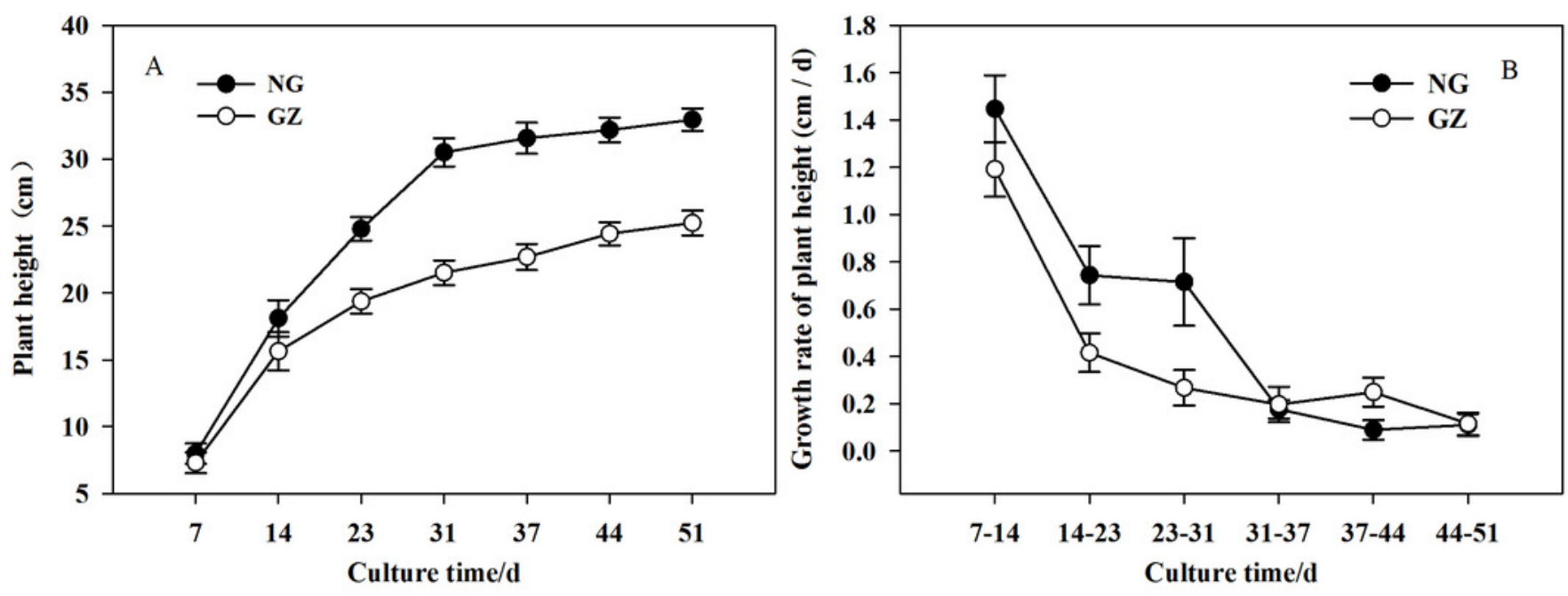


\section{Figure 2}

Effects of historical grazing on leaf length (A), width (B), area (C), and aboveground biomass (D) in offspring of Leymus chinensis (Mean $\pm \mathrm{SE}$ ) .

NG, Ungrazed; GZ, Grazed. Different asterisks indicate a significant difference between the grazing and ungrazing treatments. $*, p<0.05 ; * *, p<0.01 ; * * *, p<0.001$. The same below.
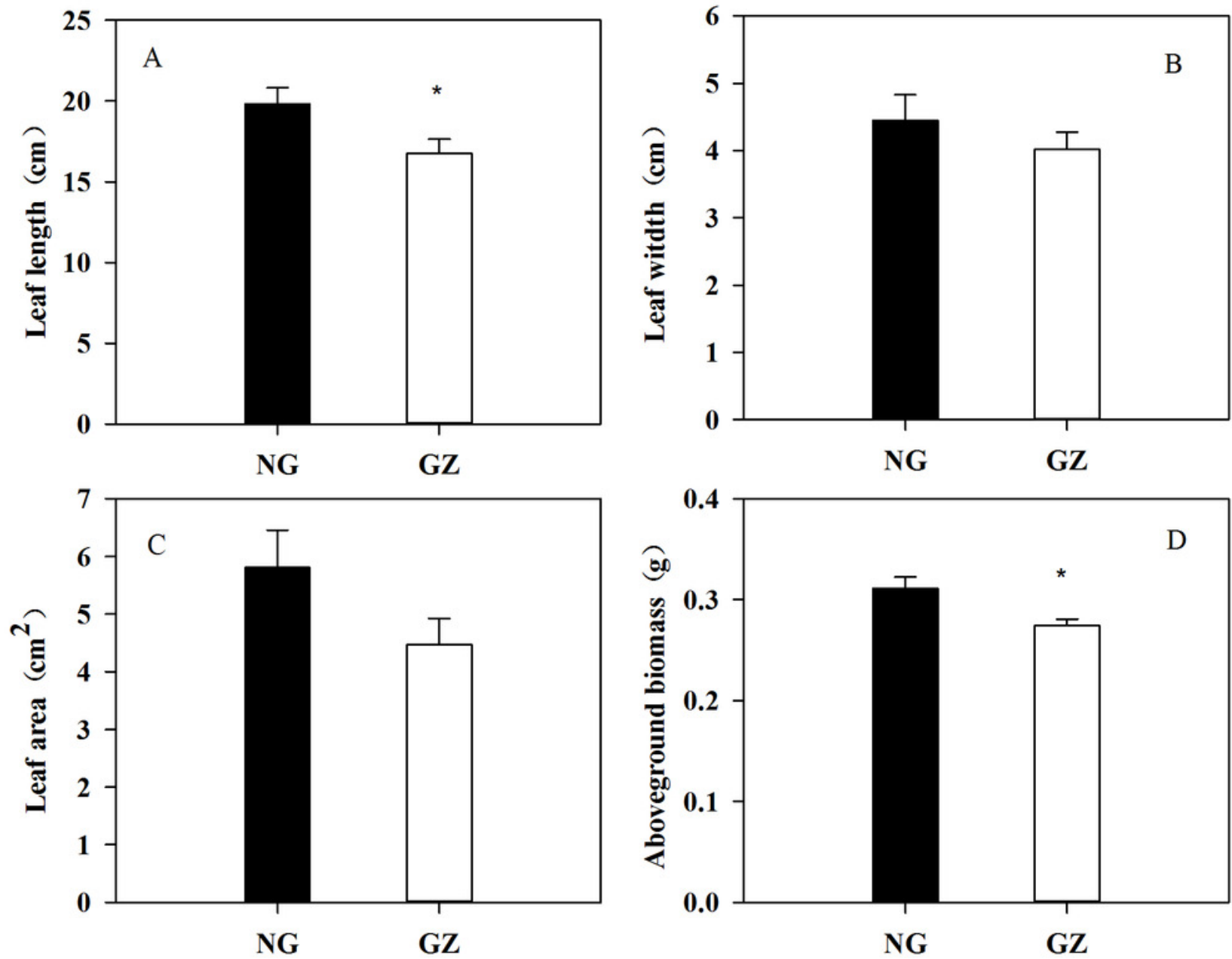
Figure 3

Effects of historical grazing on leaf photosynthetic characteristics in progeny plants (Mean \pm SE) .

$P_{N}$, net photosynthetic rate; $g s$, stomatal conductance; $C i$, intercellular carbon dioxide concentration; E, transpiration rate; WUE, water use efficiency. NG, Ungrazed; GZ, Grazed.
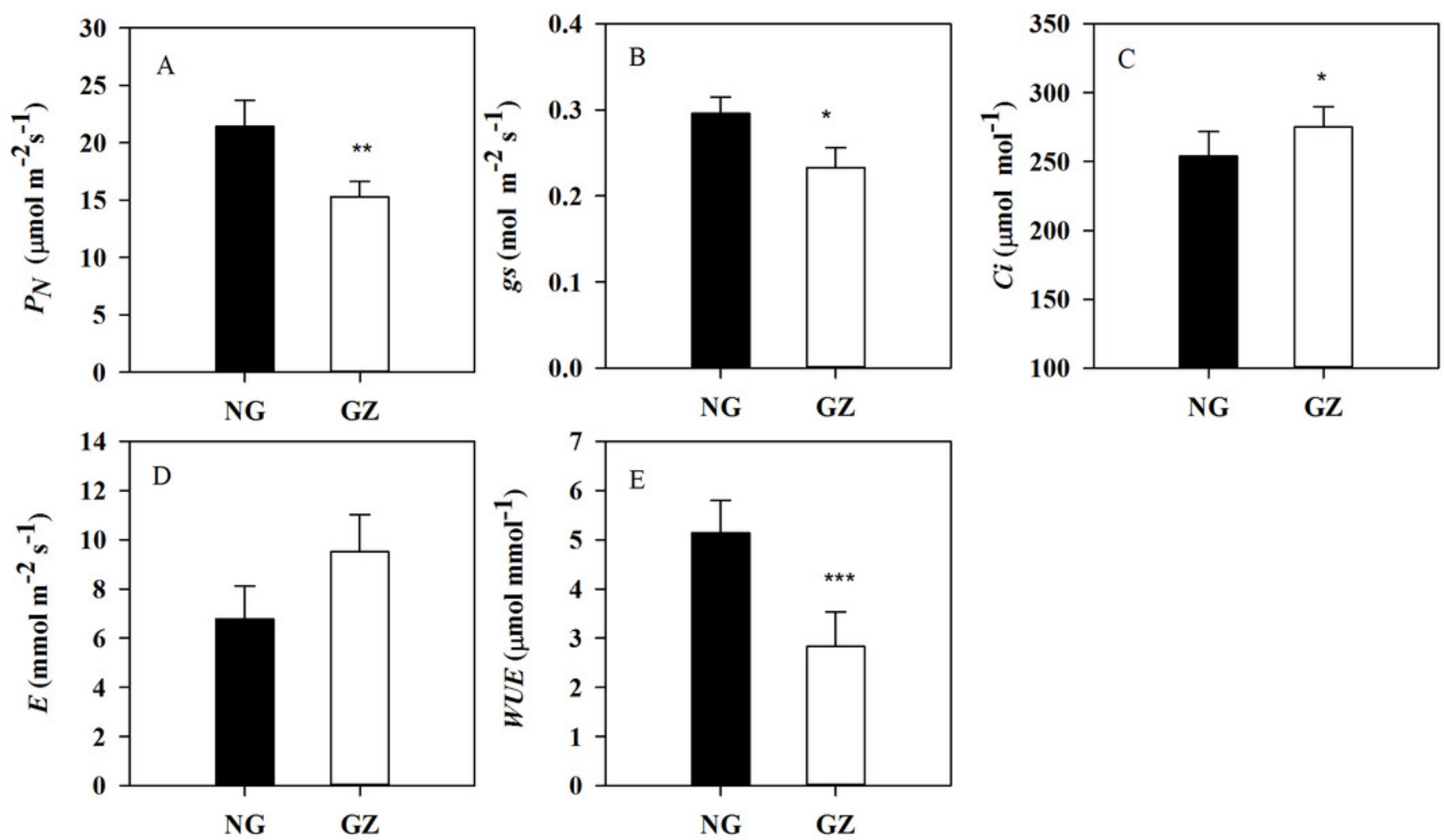
Figure 4

Effects of historical grazing on stomatal characteristics (stomatal density, length and width) of adaxial $(A, B, C)$ and abaxial $(D, E, F)$ leaf surfaces in progeny plants of Leymus Chinensis (Mean $\pm \mathrm{SE}$ ).

NG, Ungrazed; GZ, Grazed. 

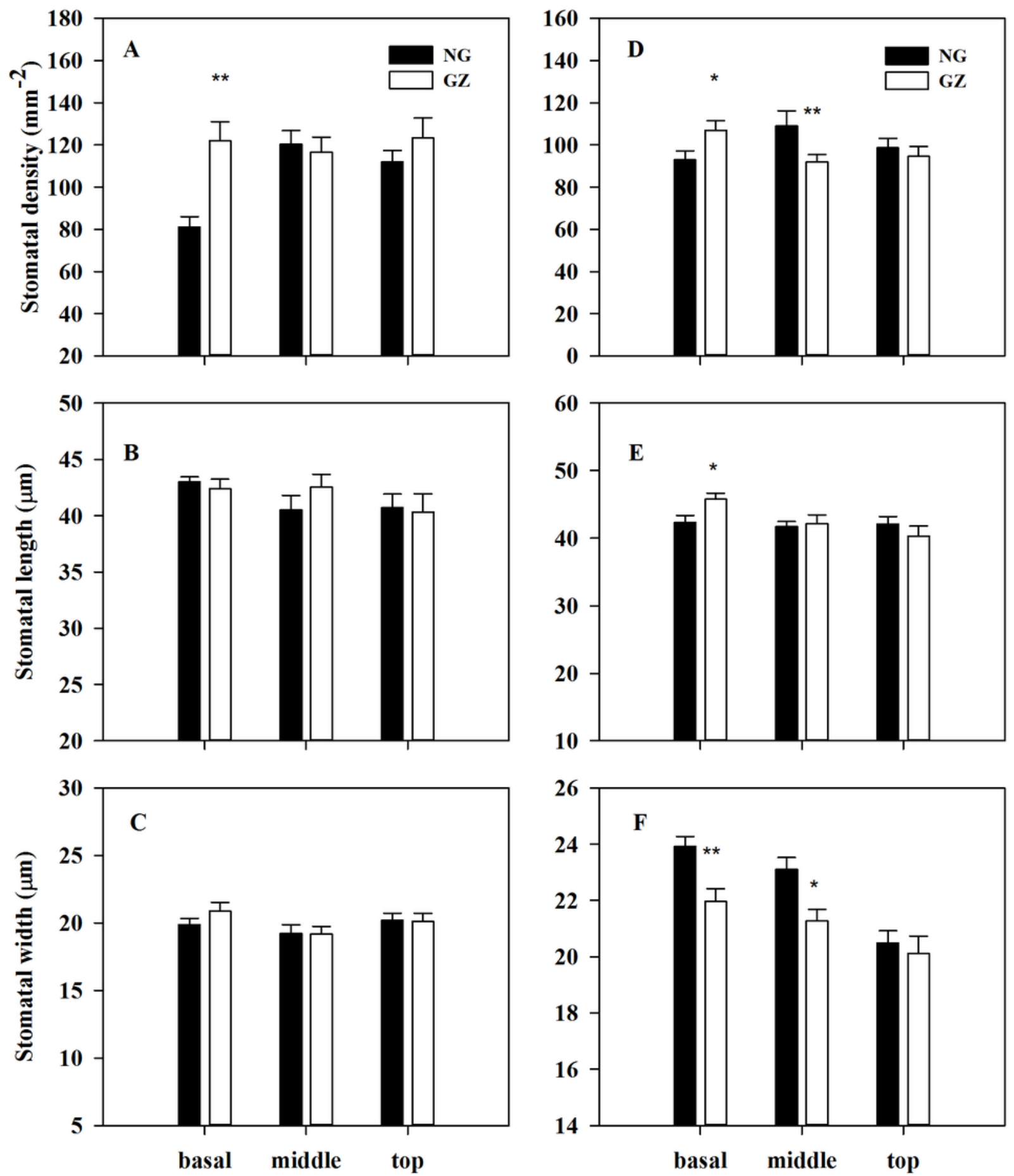
Figure 5

Differences in expression of genes related to stomatal regulation of progeny plants developed from overgrazed and ungrazed Leymus chinensis respectively.

NG, Ungrazed; GZ, Grazed.
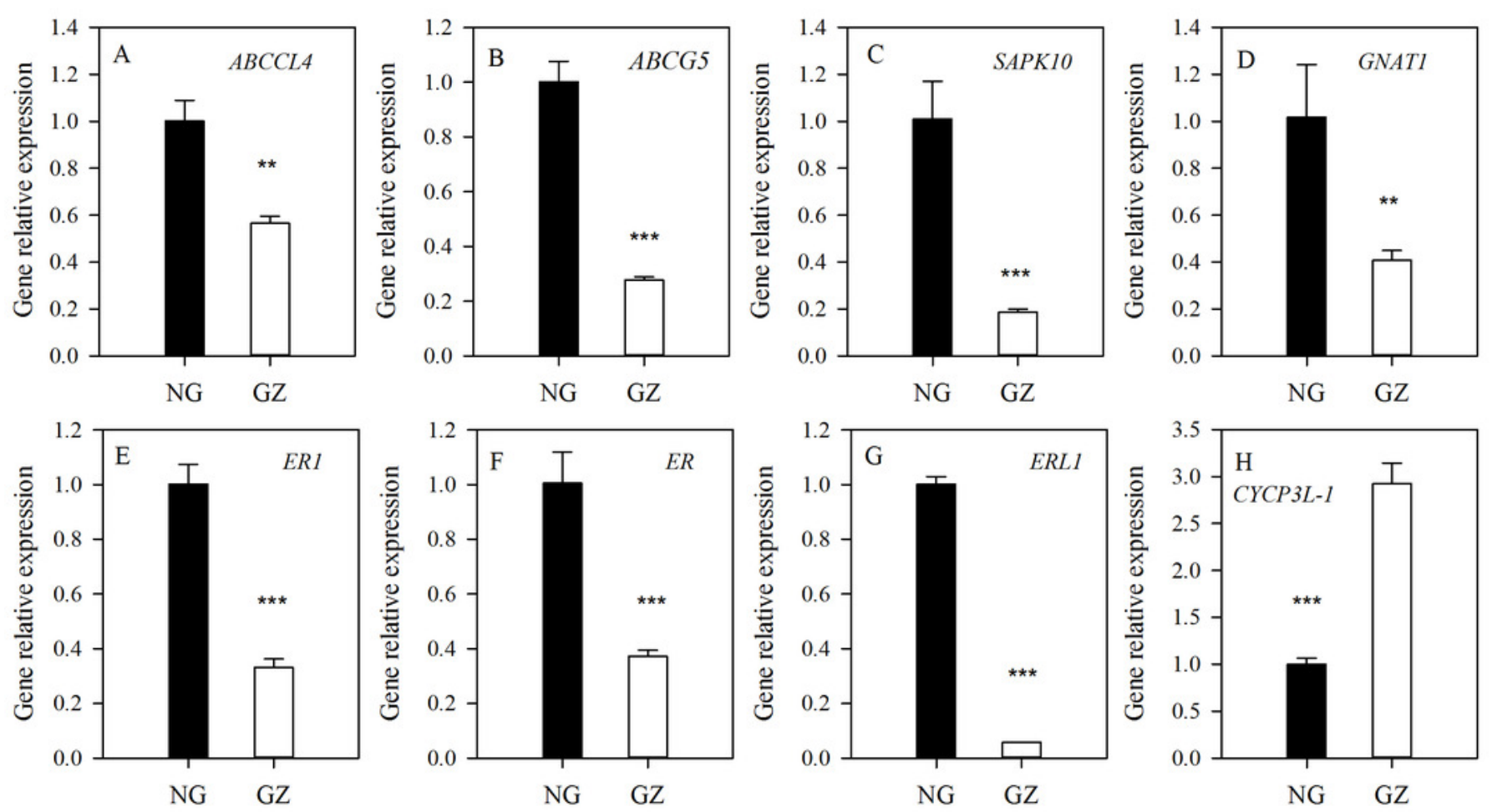
Figure 6

Schematic diagram illustrates the regulation pathway of historical overgrazing-induced dwarf phenotype in Leymus chinensis.
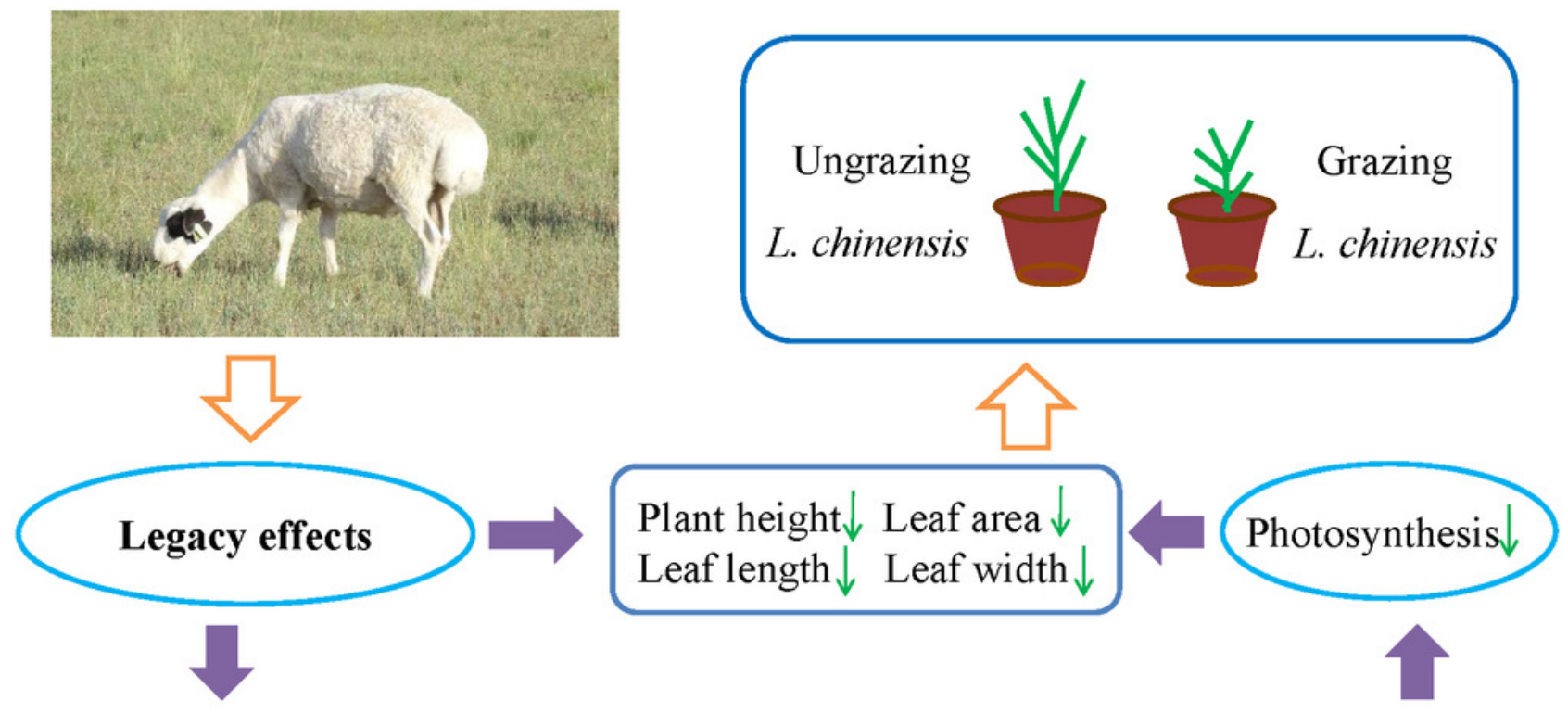

Plant height $\downarrow$ Leaf area $\downarrow$ Leaf length $\downarrow$ Leaf width $\downarrow$

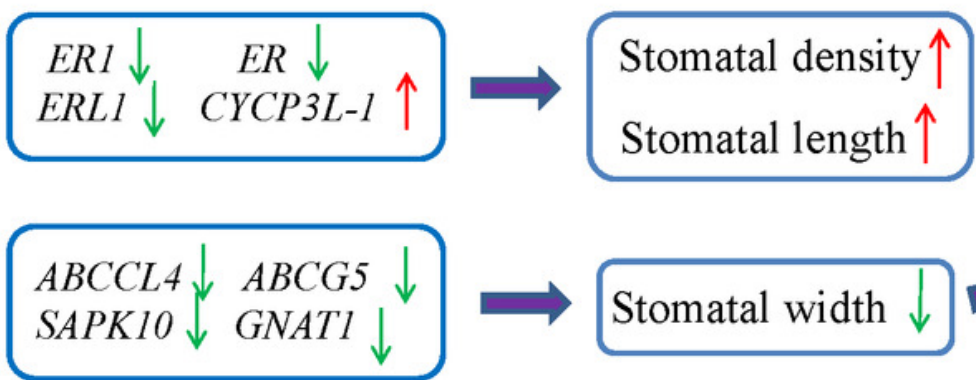

Stomatal conductance $\downarrow$ 


\section{Table 1 (on next page)}

The expression of genes related stomatal development and regulation

Note: Up, up-regulation; Down, down-regulation. 
1 Table 1:

2 The expression of genes related stomatal development and regulation

\begin{tabular}{|c|c|c|c|}
\hline Gene No. & Annotation & Abbreviation & Function \\
\hline >c118857.graph_c0 & $\begin{array}{l}\text { ABC transporter C family member 4-like } \\
\text { (LOC109773936) }\end{array}$ & $A B C C L 4$ & Stomatal movements \\
\hline >c92989.graph_c0 & $\begin{array}{l}\text { ABC transporter G family member } 5 \\
\text { (LOC109768344) }\end{array}$ & $A B C G 5$ & Stomatal movements \\
\hline >c89156.graph_c0 & $\begin{array}{l}\text { serine/threonine-protein kinase SAPK10 } \\
\text { (LOC109758947) }\end{array}$ & SAPK10 & Stomatal movements \\
\hline >c103838.graph_c0 & $\begin{array}{l}\text { guanine nucleotide-binding protein alpha-1 } \\
\text { subunit (LOC112875249) }\end{array}$ & GNAT1 & Stomatal movements \\
\hline >c128305.graph_c0 & $\begin{array}{l}\text { receptor-like serine/threonine protein kinase } \\
1 \text { (ER1) gene (JQ599260) }\end{array}$ & ERl & Stomatal development \\
\hline >c93997.graph_c0 & $\begin{array}{l}\text { LRR receptor-like serine/threonine-protein } \\
\text { kinase ERECTA (LOC109740979) }\end{array}$ & $E R$ & Stomatal development \\
\hline >c113269.graph_c0 & $\begin{array}{l}\text { LRR receptor-like serine/threonine-protein } \\
\text { kinase ERL1 (LOC109783729) }\end{array}$ & ERL1 & Stomatal development \\
\hline >c134962.graph_c0 & cyclin-P3-1-like (LOC109778329) & $C Y C P 3 L-1$ & Stomatal development \\
\hline
\end{tabular}

3 Note: Up, up-regulation; Down, down-regulation. 